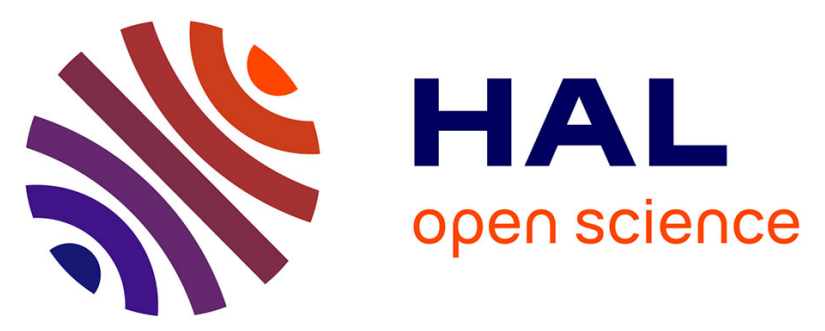

\title{
Development and external validation of a pathological nodal staging score for patients with clear cell renal cell carcinoma
}

Malte Rieken, Stephen A Boorjian, Luis Kluth, Umberto Capitanio, Alberto Briganti, R. Houston Thompson, Bradley C. Leibovich, Laura-Maria Krabbe, Vitaly Margulis, Jay D. Raman, et al.

\section{To cite this version:}

Malte Rieken, Stephen A Boorjian, Luis Kluth, Umberto Capitanio, Alberto Briganti, et al.. Development and external validation of a pathological nodal staging score for patients with clear cell renal cell carcinoma. World Journal of Urology, In press, 10.1007/s00345-018-2555-5 . hal-01990076

\section{HAL Id: hal-01990076 https://hal.sorbonne-universite.fr/hal-01990076}

Submitted on 22 Jan 2019

HAL is a multi-disciplinary open access archive for the deposit and dissemination of scientific research documents, whether they are published or not. The documents may come from teaching and research institutions in France or abroad, or from public or private research centers.
L'archive ouverte pluridisciplinaire HAL, est destinée au dépôt et à la diffusion de documents scientifiques de niveau recherche, publiés ou non, émanant des établissements d'enseignement et de recherche français ou étrangers, des laboratoires publics ou privés. 


\title{
Development and external validation of a pathological nodal staging score for patients with clear cell renal cell carcinoma
}

\author{
Malte Rieken ${ }^{1,2,14}$. Stephen A. Boorjian ${ }^{3}$ Luis A. Kluth ${ }^{2,4}$. Umberto Capitanio ${ }^{5}$ - Alberto Briganti ${ }^{5}$. \\ R. Houston Thompson ${ }^{3} \cdot$ Bradley C. Leibovich $^{3} \cdot$ Laura-Maria Krabbe $^{6} \cdot$ Vitaly Margulis $^{6} \cdot$ Jay D. Raman $^{7}$. \\ Mikhail Regelman ${ }^{7} \cdot$ Pierre I. Karakiewicz $^{8} \cdot$ Morgan Rouprêt $^{2,9} \cdot$ Mohammad Abufaraj $^{1,10}$ (1) Beat Foerster ${ }^{1,11}$. \\ Mithat Gönen ${ }^{12}$. Shahrokh F. Shariat ${ }^{1,2,6,13}$
}

\begin{abstract}
Objectives To develop and externally validate a model that quantifies the likelihood that a pathologically node-negative patient with clear cell renal cell carcinoma (cRCC) has, indeed, no lymph node metastasis (LNM).

Patients and methods Data from 1389 patients treated with radical nephrectomy (RN) and lymph node dissection (LND) were analyzed. For external validation, we used data from 2270 patients in the Surveillance, Epidemiology and End Results (SEER) database. We estimated the sensitivity of pathologic nodal staging using a beta-binomial model and developed a pathological nodal staging score (pNSS), which represents the probability that a patient is correctly staged as node negative as a function of the number of examined lymph nodes (LNs).

Results The mean and median number of LNs removed were 7.0 and 5.0 (standard deviation, SD 6.6; interquartile range, IQR 7.0) in the development cohort and 5.6 and 2.0 (SD 8.6, IQR 5.0) in the validation cohort, respectively. The probability of missing a positive LN decreased with increasing number of LNs examined. In both the validation and the development cohort, the number of LNs needed for correctly staging a patient as node negative increased with higher pathological tumor stage and Fuhrman grade.

Conclusions The number of examined LNs needed for adequate nodal staging in cRCC depends on pathological tumor stage and Fuhrman grade. We developed here and then externally validated a pNSS, which could help to refine patient counseling, decision-making regarding risk-stratified surveillance regimens and inclusion criteria for clinical trials of adjuvant therapy.
\end{abstract}

Keywords Clear cell renal cell carcinoma · Radical nephrectomy · Lymph node dissection · Lymph node metastasis · Validation · SEER

Shahrokh F. Shariat

sfshariat@gmail.com

1 Department of Urology, Medical University of Vienna, Währinger Gürtel 18-20, 1090 Vienna, Austria

2 Department of Urology, Weill Cornell Medical College, New York-Presbyterian Hospital, New York, NY, USA

3 Department of Urology, Mayo Medical School and Mayo Clinic, Rochester, MN, USA

4 Department of Urology, University Medical Center Hamburg-Eppendorf, Hamburg, Germany

5 Department of Urology, Urological Research Institute, San Raffaele Scientific Institute, Milan, Italy

6 Department of Urology, University of Texas Southwestern Medical Center, Dallas, TX, USA

7 Division of Urology, Penn State Milton S. Hershey Medical Center, Hershey, PA, USA
8 Department of Urology, University of Montreal, Montreal, QC, Canada

9 Department of Urology, Pitié-Salpétrière, Assistance-Publique Hôpitaux de Paris, Faculté de Médecine Pierre et Marie Curie, University Paris VI, Paris, France

10 Division of Urology, Department of Special Surgery, Jordan University Hospital, The University of Jordan, Amman, Jordan

11 Department of Urology, Kantonsspital Winterthur, Winterthur, Switzerland

12 Department of Epidemiology and Biostatistics, Memorial Sloan-Kettering Cancer Center, New York, NY, USA

13 Karl Landsteiner Institute of Urology and Andrology, Vienna, Austria

14 University of Basel, Basel, Switzerland 


\section{Introduction}

Radical nephrectomy (RN) or nephron-sparing surgery remains the mainstay of therapy to achieve cure in patients with localized renal cell carcinoma (RCC) [1]. Despite surgical treatment, a non-negligible fraction of patients with localized RCC experience disease recurrence [2]. Among other factors, lymph node metastasis (LNM) has been demonstrated to adversely impact oncologic outcomes in patients with RCC [3,4]. As the presence of LNM has implications for follow-up scheduling and potentially adjuvant treatment with targeted therapies, accurate nodal staging appears necessary.

Currently available imaging technologies are limited by a detection threshold for LNM; thus, lymph node dissection (LND) remains the most accurate form of nodal staging in patients with localized RCC [5]. However, no clear consensus exists regarding the indications and the extent of LND at RN [5]. Indeed, the anatomic unpredictability of lymphatic outflow in RCC and the early manifestation of distant metastases without LNM have hampered efforts to define a uniformly accepted extent of LND in RCC [6-8]. In recent years, efforts have been made to estimate the presence of LNM in patients with RCC and the number of LNs necessary to achieve adequate nodal staging [9-11].

We developed a methodology (pathological nodal staging score, pNSS) to calculate the probability that a patient with pathologic node-negative status at surgery does in fact not have LNM, using the number of examined LNs and established clinicopathologic features of a given tumor type [12-15]. The aim of the current study, then, was to develop and externally validate such a prognostic model for patients with clear cell renal cell carcinoma (cRCC) treated with RN. Toward this aim, we developed the pNSS using a multicenter cohort of patients who underwent RN and LND for cRCC. For validation, we used a contemporary, population-based cohort by accessing the Surveillance, Epidemiology and End Results (SEER) database. Use of SEER allowed us to test whether the pNSS developed in multicenter individual dataset is generalizable and reproducible in a population-based dataset.

\section{Materials and methods}

\section{Patient selection and data collection}

The development cohort comprised 1389 patients who underwent open $(n=1286,92.6 \%)$ or laparoscopic $(n=103,7.4 \%) \mathrm{RN}$ and LND for clinically localized cRCC at five international academic centers between
1970 and 2012. The indications and extent of LND were at the surgeon's discretion. No patient received preoperative radiotherapy, immunotherapy or targeted therapy. No patient had clinically evident distant metastatic disease at the time of RN. Institutional review boards approved the study, with all participating sites providing the necessary institutional data sharing agreements beforehand.

For the contemporary validation cohort $(n=2279)$, we used Surveillance, Epidemiology and End Results (SEER) registry data from 2004 to 2009 . By the end of the study period, the registry captured approximately $28 \%$ of the US population, and is considered to be representative of the general population. Patients who underwent RN for kidney cancer (code C 64.9) were identified. Inclusion criteria consisted of those patients having a diagnosis of cRCC, and documentation of the number of LNs examined as well as the number of pathologically positive LNs. Patients were excluded from analyses when Fuhrman grade or tumor stage was unknown.

\section{Pathological evaluation}

All surgical specimens were processed according to standard pathological procedures as previously described [1]. Genitourinary pathologists determined tumor stage, which was in the development cohort reassigned according to the 2009 tumor node metastasis (TNM) classification [16]. In SEER, pathological tumor stage category was derived from collaborative staging data elements for all cases, consistent with the 2002 TNM classification [17]. To account for these different staging classifications in the development and validation cohort, pathological stage $\mathrm{T} 1 / 2$ and $\mathrm{T} 3 / 4$ patients were analyzed combined together. The Fuhrman classification was used for the assessment of nuclear grade [18]. Histologic subtypes were assigned according to the $2004 \mathrm{WHO}$ classification [19].

\section{Statistical analysis}

\section{Overview}

We applied a methodology similar to that previously described [12-15], to build a pNSS each for the development and validation cohorts. The primary endpoint was the probability of incorrect nodal staging as a function of the number of examined LNs $(n)$ [12-15]. Although true nodal status is unascertainable, the information from LN-positive patients can be used to determine if the number of examined LNs and those of negative LNs are sufficient to classify a patient as truly LN negative. For example, consider a patient with $n$ large and $k$ small, but positive ( $k=$ number of positive LNs from patients with LNM): if less than $n$ LNs had been examined there would be a chance that this patient would have been incorrectly 
deemed LN negative. Conversely, for a patient with small $n$ and large $k$, even with fewer examined LNs, it is unlikely that nodal disease would have been missed. Hence, the data from LN-positive patients are used to interpret the data for the LNnegative patients. The probability that a $\mathrm{LN}$-negative patient has LNM can be computed using the following algorithm: compute the probability of missing a positive node (sensitivity), compute the prevalence of node-positive status, and compute the nodal staging score from sensitivity and prevalence [12-15].

\section{Probability of missing a positive LN}

The probability of missing a positive LN (one minus the sensitivity) in pNO patients is inherent to the process of pathological detection and as such depends on the number of examined LNs but not on patient characteristics [12-15]. We used a $\beta$-binomial model for this purpose, allowing for heterogeneity in the intensity of nodal spread across the patients [12-15].

\section{Estimation of prevalence of nodal disease}

The observed prevalence (called apparent prevalence hereafter) is underestimated and needs to be adjusted for the false negatives [12-15]. This was done in two steps. The first step invokes assumption one and estimates $\# \mathrm{FN}_{k}$ as a function of $k$, which is the number of positive LNs from patients with LN involvement:

$\# \mathrm{FN}_{k}=\frac{\left[1-P\left(\mathrm{FN}_{k}\right)\right] \times \# \mathrm{TP}_{k}}{P\left(\mathrm{FN}_{k}\right)}$,

where $\# \mathrm{TP}_{k}$ is the number of true positives for a given $k$. Since prevalence is not a function of $k$, the second step obtains the adjusted prevalence by averaging over $k$ :

Prev $=\frac{\sum_{k}\left(\mathrm{FN}_{k}+\mathrm{TP}_{k}\right)}{\sum_{k}\left(\mathrm{FN}_{k}+\mathrm{TP}_{k}+\mathrm{TN}_{k}\right)}$.

Estimation of prevalence is stratified by $\mathrm{T}$ stage for pNSS, but this is not explicitly noted in the above formula to avoid cumbersome notation.

\section{Nodal staging score}

Adequate staging was assessed by computing the NSS, the probability that a pathologically LN-negative patient is indeed free of nodal disease:

$\mathrm{NSS}=\frac{1-\text { Prev }}{1-\text { Prev }+\left[\text { Prev } \times P\left(\mathrm{FN}_{k}\right)\right]}$.

\section{Confidence intervals}

Precision of the reported estimates was assessed by creating 1000 bootstrap samples from the entire data set and replicating the estimation process. The 2.5 th and the 97.5th quartiles were used as the lower and upper $95 \%$ confidence bounds for the corresponding estimates.

\section{Validation of the development model}

After development of the pNSS model in the data from the development cohort, we next compared these findings to those from the validation cohort. We compared the two populations using the Chi-square test to evaluate the association between categorical variables. Differences in variables with a continuous distribution across categories were assessed using the Kruskal-Wallis test. In addition, we compared the probabilities of missing a LN either based on the number of LNs removed/examined or combining the number of LNs removed/examined with pathological stage and Fuhrman grade. All statistical analyses were performed using SAS Version 9.2.

\section{Results}

\section{Clinicopathologic characteristics in the development and validation cohorts}

Table 1 shows the clinicopathologic characteristics of the development $(n=1389)$ and the validation cohorts $(n=2279)$. LNM was detected in $14.3 \%(n=198)$ of the patients in the development cohort compared to $10.0 \%$ $(n=227)$ in the validation cohort $(p<0.001)$. The median number of LNs removed in patients with LNM was significantly higher in the development cohort compared to the validation cohort ( 7.0 vs. $2.0, p<0.001)$.

\section{Probability of missing a positive LN}

Using our model, the beta-binomial parameters were estimated to be 1.01 (95\% CI 0.72-1.29) and 0.53 (95\% CI $0.34-0.72$ ) in the validation cohort. We assessed the probability of LNM (one minus the sensitivity) as a function of the number of LNs examined (Fig. 1). In both the development and validation cohorts, the probability of missing LNM decreases with an increasing number of LNs examined (Fig. 1). When compared to the probabilities of missing a LN in the development cohort, fewer LNs were 
Table 1 Clinicopathologic characteristics of 1389 patients in the development cohort and 2279 patients in the validation cohort who underwent radical nephrectomy and lymph node dissection for clinically localized clear cell renal cell carcinoma

\begin{tabular}{llll}
\hline Characteristics & $\begin{array}{l}\text { Development } \\
\text { cohort } \\
(n=1389)\end{array}$ & $\begin{array}{l}\text { Validation cohort } \\
(n=2279)\end{array}$ & $p$ value \\
& & \\
\hline
\end{tabular}

Age (years)

Mean (SD)

$60.8(11.4)$

$60.4(12.0)$

0.31

Median (IQR)

$62.0(16)$

$60.0(17)$

Gender $(n, \%)$

$\begin{array}{llc}\text { Male } & 967(69.6) & 1466(64.3) \\ \text { Female } & 422(30.4) & 813(35.7)\end{array}$

0.001

Pathological tumor stage $(n, \%)$

$\begin{array}{lccc}\text { pT1 } & 418(30.1) & 781(34.3) & <0.001 \\ \text { pT2 } & 236(17.0) & 500(21.9) & \\ \text { pT3 } & 681(49.0) & 966(42.4) & \\ \text { pT4 } & 54(3.9) & 32(1.4) & \end{array}$

Fuhrman grade $(n, \%)$

$\begin{array}{lll}\text { Grade I } & 104(7.5) & 177(7.8) \\ \text { Grade II } & 544(39.2) & 972(42.7) \\ \text { Grade III } & 589(42.4) & 862(37.8) \\ \text { Grade IV } & 152(10.9) & 268(11.8)\end{array}$

Pathological tumor stage/Fuhrman grade combined $(n, \%)$

$\begin{array}{llll}\text { pT1/2, grade I/II } & 455(32.8) & 829(36.4) & <0.001 \\ \text { pT1/2, grade III/ } & 199(14.3) & 452(19.8) & \\ \text { IV } & & & \\ \text { pT3/4, grade I/II } & 193(13.9) & 320(14.0) \\ \text { pT3/4, grade III/ } & 542(39.0) & 678(29.7)\end{array}$

Lymph node metastasis ( $n, \%)$

$\begin{array}{lrrr}\text { No } & 1191(85.7) & 2052(90.0) & <0.001 \\ \text { Yes } & 198(14.3) & 227(10.0) & \end{array}$

Number of examined lymph nodes (all patients)

$\begin{array}{lll}\text { Mean (SD) } & 7.0(6.6) & 5.6(8.4) \\ \text { Median (IQR) } & 5.0(7.0) & 2.0(5.0)\end{array}$

Number of examined lymph nodes (node-negative patients)

$\begin{array}{llll}\text { Mean (SD) } & 6.5(5.8) & 5.6(8.6) & <0.001 \\ \text { Median (IQR) } & 5.0(7.0) & 2.0(5.0) & \end{array}$

Number of examined lymph nodes (node-positive patients)

$\begin{array}{lccc}\text { Mean (SD) } & 10.0(9.6) & 5.3(6.4) & <0.001 \\ \text { Median (IQR) } & 7.0(10) & 2.0(6.0) & \end{array}$

Number of positive lymph nodes (node-positive patients)

\begin{tabular}{llll} 
Mean (SD) & $3.9(4.3)$ & $2.4(2.4)$ & $<0.001$ \\
Median (IQR) & $2.0(4.0)$ & $1.0(2.0)$ & \\
\hline
\end{tabular}

needed in the validation cohort to reach the same level of probability; however, these differences were not statistically significant (all $p$ values $>0.05$ ).

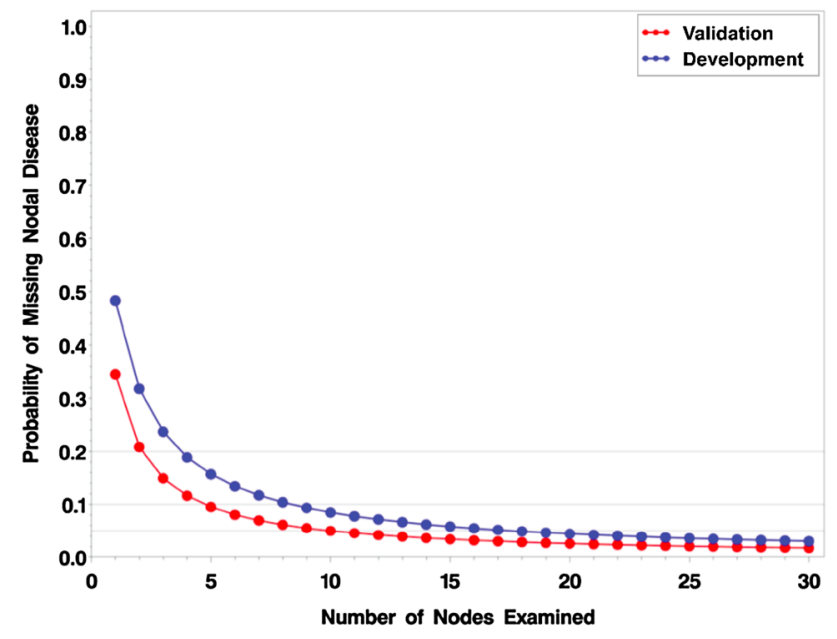

Fig. 1 Probability of missing nodal disease as a function of nodes examined in 1389 patients in the development cohort and 2279 patients in the validation cohort who were treated with radical nephrectomy and lymphadenectomy for clinically localized clear cell renal cell carcinoma

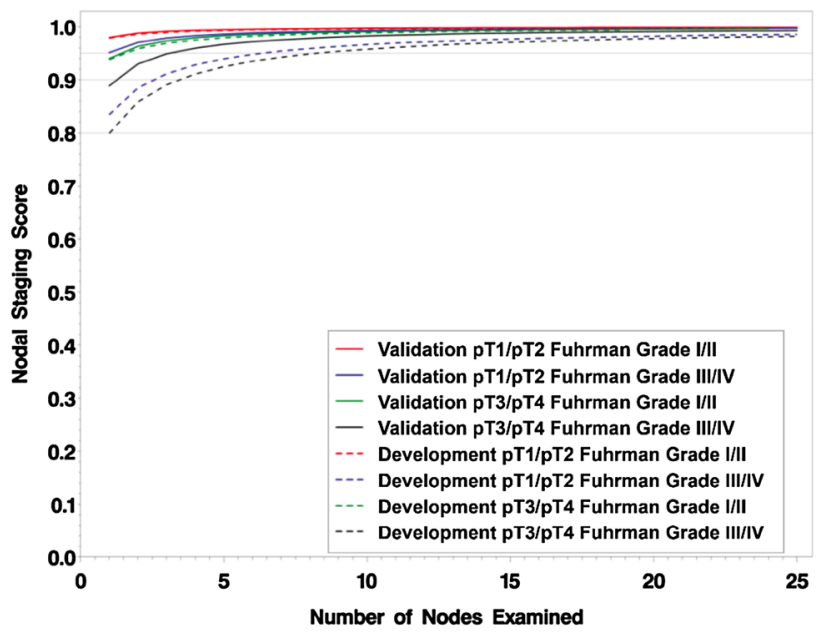

Fig. 2 Pathologic nodal staging scores: sensitivity of the pathologic evaluation of nodal disease stratified by pathological tumor stage in combination with Fuhrman grade of 1389 patients in the development cohort and 2279 patients in the validation cohort who were treated with radical nephrectomy and lymphadenectomy for clinically localized clear cell renal cell carcinoma. The vertical axis is the probability of missing nodal disease (one minus sensitivity); the horizontal axis is the number of examined nodes

\section{Pathological nodal staging score}

Figure 2 and Table 2 show the pNSS in the development and validation cohort. In patients with pT1/2 and Fuhrman grade I/II tumors, in both the development and validation cohorts, the examination of only one $\mathrm{LN}$ was sufficient to achieve a likelihood of more than $95 \%$ to predict correct 
Table 2 Pathologic nodal staging score for selected values of number of nodes removed of 1389 patients in the development cohort and 2279 patients in the validation cohort who underwent radical nephrectomy and lymph node dissection for clinically localized clear cell renal cell carcinoma

\begin{tabular}{|c|c|c|c|c|c|c|c|c|}
\hline No. of examined nodes & 1 & 3 & 5 & 8 & 10 & 12 & 15 & 20 \\
\hline \multicolumn{9}{|c|}{ pT1/2, Fuhrman grade I/II } \\
\hline Development cohort & 97.8 & 98.9 & 99.3 & 99.5 & 99.6 & 99.7 & 99.7 & - \\
\hline Validation cohort & 98.0 & 99.1 & 99.4 & 99.6 & 99.7 & 99.7 & 99.8 & 99.8 \\
\hline \multicolumn{9}{|c|}{ pT1/2, Fuhrman grade III/IV } \\
\hline Development cohort & 83.4 & 91.1 & 93.9 & 95.9 & 96.6 & 97.1 & 97.7 & - \\
\hline Validation cohort & 95.1 & 97.8 & 98.6 & 99.1 & 99.3 & 99.4 & 99.5 & 99.6 \\
\hline \multicolumn{9}{|c|}{ pT3/4, Fuhrman grade I/II } \\
\hline Development cohort & 93.8 & 96.9 & 97.9 & 98.6 & 98.9 & 99.0 & 99.2 & 99.4 \\
\hline Validation cohort & 94.0 & 97.3 & 98.3 & 98.9 & 99.1 & 99.2 & 99.4 & 99.5 \\
\hline \multicolumn{9}{|c|}{ pT3/4, Fuhrman grade III/IV } \\
\hline Development cohort & 79.9 & 89.0 & 92.4 & 94.9 & 95.8 & 96.4 & 97.1 & 97.7 \\
\hline Validation cohort & 88.9 & 94.9 & 96.7 & 97.8 & 98.2 & 98.5 & 98.8 & 99.1 \\
\hline
\end{tabular}

Table 3 Apparent and corrected prevalences of lymph node metastasis in the original cohort of 1389 patients in the development cohort and 2279 patients in the validation cohort who underwent radical nephrectomy and lymph node dissection for clinically localized clear cell renal cell carcinoma

\begin{tabular}{lcc}
\hline & $\begin{array}{l}\text { Apparent preva- } \\
\text { lence }\end{array}$ & $\begin{array}{l}\text { Corrected } \\
\text { prevalence }\end{array}$ \\
\hline All cases & & \\
$\quad$ Development cohort & 18.0 & 22.8 \\
$\quad$ Validation cohort & 11.9 & 15.2 \\
pT1/2, Fuhrman grade I/II & & \\
$\quad$ Development cohort & 3.4 & 4.4 \\
$\quad$ Validation cohort & 4.4 & 5.7 \\
pT1/2, Fuhrman grade III/IV & & \\
$\quad$ Development cohort & 21.3 & 29.2 \\
$\quad$ Validation cohort & 9.9 & 13.0 \\
pT3/4, Fuhrman grade I/II & & \\
$\quad$ Development cohort & 10.1 & 12.0 \\
$\quad$ Validation cohort & 12.0 & 15.6 \\
pT3/4, Fuhrman grade III/IV & & 34.2 \\
$\quad$ Development cohort & 27.5 & 26.6 \\
Validation cohort & 21.4 & \\
\hline
\end{tabular}

pathologic nodal status. Meanwhile, three LNs were sufficient to achieve a likelihood of more than $95 \%$ to predict correct pathologic nodal status in patients with pT3/4 and Fuhrman grade I/II tumors. In contrast, the number of LNs examined to achieve a certain probability of being free from LNM needed to be higher in patients with Fuhrman grade III/IV tumors. Bootstrap CIs for all the estimates were within $1 \%$ (in absolute terms) of the estimates (data not shown). Significant differences in the probabilities between the development and validation cohorts were detected in patients with Fuhrman grade III/IV tumors $(p<0.001)$ (Fig. 2).

\section{Apparent and corrected prevalence of nodal disease}

The apparent and corrected prevalences of nodal metastasis stratified by pathologic T stage and Fuhrman grade in both cohorts are reported in Table 3. Underestimation of prevalence due to false negatives was observed for all examined subgroups of patients. Statistically significant differences were found between the validation and the development cohorts with regard to apparent and corrected prevalences of nodal diseases in all cases $(p<0.001)$, pT $1 / 2$ and Fuhrman grade I/II $(p<0.001)$, pT $1 / 2$ and Fuhrman grade III/ IV $(p<0.001)$, as well as pT3/4 and Fuhrman grade III/IV cases $(p<0.001)$.

\section{Discussion}

The presence of LNM is a strong predictor of adverse outcomes in patients with cRCC undergoing RN $[1,3]$. Indeed, RCC patients with regional LNM have a limited 5-year survival [5]. Accordingly, then, such patients with cRCC may be considered for adjuvant targeted therapies [20]. Knowledge of the lymph node status is important to allow proper risk estimation [5, 21-23] for counseling and followup scheduling as well as timely consideration of systemic therapy. Therefore, to estimate the probability that a cRCC patient with pathologic node-negative status at RN truly has no LNM, we developed and validated a pNSS. In both the development and the validation cohorts, the probability of missing LNM decreased with an increasing number of LNs examined. This is in line with previous studies, which aimed to identify a minimum number of LNs that need to be removed to obtain satisfactory nodal staging at time of $\mathrm{RN}$ [10]. In patients with RCC who underwent LND, a statistically significant correlation between the number of LNs removed and the percentage of nodal involvement could be shown $[24,25]$. While the absolute number of LNs removed 
is important in estimating the probability of missing LNM, standard clinicopathologic features need to be taken into consideration as well.

We found that the number of LNs needed for appropriate nodal staging is associated with higher pathological tumor stage and Fuhrman grade. Our findings confirm previous studies showing that the proportion of patients having LNM increased proportionally with more aggressive disease [4]. Specifically, in a series of 1652 patients undergoing RN for cM0 cRCC, multivariable analysis demonstrated that the presence of Fuhrman grade 3 or 4, sarcomatoid component, tumor size larger than $10 \mathrm{~cm}$, tumor stage pT3 or pT4, and coagulative tumor necrosis were independent predictors of LNM [4]. A preoperative nomogram predicting the presence of LNM and/or the probability of LN progression during follow-up has been developed as well [9], although the model still awaits prospective external validation [9]. Despite improved imaging, LND remains the most reliable form of nodal staging in patients with RCC. According to guidelines, LND is not recommended in localized tumor without clinical evidence of LNM [1]. In contrast, in patients with palpable or CT-detected enlarged lymph nodes, resection of the affected LNs should be performed to obtain adequate staging information and local control $[1,12]$. Of note, our study included only patients without clinical evidence of LNM.

The accuracy of nodal staging achieved with a given number of LNs removed differed between the development and validation cohorts in patients with Fuhrman grade III/IV regardless of pathologic stage. These differences highlight one limitation of our model, that is, as the model is based on the actual number of LNs removed in each given cohort of patients, the number of LNs to be examined tends to be higher in the cohort of patients with the higher median number of LNs removed. These differences are not surprising as SEER contains data of both academic and community centers.

We developed and externally validated a simple probabilistic model that calculates the probability of freedom from occult LNM as a function of clinicopathologic parameters and number of LNs examined. Since outcome prediction based on a physician's experience alone might be subjectively influenced, several postoperative models to predict recurrence or cancer-specific mortality based on clinicopathologic features have been introduced for use in daily clinical practice [26]. Our model is a simple tool that could serve as guidance in the postoperative clinical decisionmaking regarding follow-up scheduling and administration of adjuvant therapy as part of clinical trials when possible. Based on the current lack of adequate selection criteria or biomarkers for adjuvant therapy in patients with RCC, the current study may provide a potential solution as patients with higher risk of false-negative N0 status may be candidates for adjuvant treatment in clinical trials.
Our study has several limitations. First and foremost are limitations inherent to its multicenter and retrospective designs as well as the long study period in the development cohort. We used the SEER database to validate our model in a contemporary, large cohort of patients treated both at academic and community centers. It is important to acknowledge that the performance of a LND in patients with clinically node-negative status is not yet standardized and not routinely recommended by guidelines. Currently, a LND is primarily recommended in patients with adverse clinical features including a large diameter of the primary tumor. However, the evidence on which this recommendation is based has to be regarded as weak. Nevertheless, our data may be biased as we only included patients with clinically node-negative status. The use of the contemporary SEER database as a validation cohort might introduce additional bias considering including historical cohorts in the development set. Such bias might be caused by staging and grading inaccuracies. Both datasets suffer from shortcomings such as selection criteria for LND, and variation in the use of preoperative imaging, among others. Further, the SEER database does not provide information on the extent of LND or the processing of LNs. While we were able to control for numerous potential confounders, we could not control for surgeon's and pathologist's experience, treatment decisions including surgical approach (laparoscopic vs. open), patient and surgeon preferences, as well as the anatomical template of LND. Indeed, the number of LNs examined is not an exact surrogate for the extent of LND. The low nodal yield in the validation cohort might represent the accidental $\mathrm{LN}$ finding in the hilar or perirenal fat rather than actual LND. We could not differentiate between these entities using the SEER database potentially introducing additional bias. Furthermore, the number of LNs examined is not only a factor of the extent of LND but is also dependent on the pathological evaluation and inherent differences between patients. We only included patients with cRCC and further studies will be necessary to test our model in non-cRCC cohorts. In addition, our model is built on assumptions. Although these might seem debatable, every single mathematical model and theory is built on assumptions. Prospective validation is thereby warranted to test whether the assumptions were appropriate.

\section{Conclusions}

We developed and externally validated a pNSS that estimates the likelihood of false-negative LNM with LND after RN for clinically localized cRCC. We determined that the number of examined LNs needed for adequate nodal staging depends on pathological tumor stage and Fuhrman grade. Our model may be used in patient counseling regarding postoperative 
surveillance, as well as for trial eligibility in the assessment of adjuvant therapies.

Author contributions MR contributed to data collection, management and analysis, protocol development and manuscript writing and editing. SAB helped in protocol development, data collection, and manuscript editing. LAK performed protocol development, data collection and management, and manuscript editing. RHT, BCL, L-MK, VM, BF, PIK, MR and JDR performed data collection and manuscript editing. UC, AB and MR contributed to manuscript editing. MG and MA helped in data collection and management, and manuscript editing. SFS helped in project and protocol development, data collection and analysis, and manuscript writing and editing.

\section{Compliance with ethical standards}

Conflict of interest The authors declare that they have no conflict of interest.

Ethical approval Institutional review board approval was obtained by all participating centers and provided institutional data sharing agreements in accordance with the ethical standards of the institutional and/ or national research committee.

Informed consent Informed consent was obtained from all individual participants included in the study.

\section{References}

1. Ljungberg B, Bensalah K, Bex A, Canfield S, Dabestani S, Hofmann $F$ et al (2015) EAU guidelines on renal cell carcinoma: 2014 update. Eur Urol 67(5):913-924

2. Karakiewicz PI, Suardi N, Capitanio U et al (2009) Conditional survival predictions after nephrectomy for renal cell carcinoma. J Urol 182(6):2607-2612

3. Capitanio U, Jeldres C, Patard JJ et al (2009) Stage-specific effect of nodal metastases on survival in patients with non-metastatic renal cell carcinoma. BJU Int 103(1):33-37

4. Blute ML, Leibovich BC, Cheville JC, Lohse CM, Zincke H (2004) A protocol for performing extended lymph node dissection using primary tumor pathological features for patients treated with radical nephrectomy for clear cell renal cell carcinoma. J Urol 172(2):465-469

5. Capitanio U, Becker F, Blute ML et al (2011) Lymph node dissection in renal cell carcinoma. Eur Urol 60(6):1212-1220

6. Johnsen JA, Hellsten S (1997) Lymphatogenous spread of renal cell carcinoma: an autopsy study. J Urol 157(2):450-453

7. Lughezzani G, Capitanio U, Jeldres C et al (2009) Prognostic significance of lymph node invasion in patients with metastatic renal cell carcinoma: a population-based perspective. Cancer 115(24):5680-5687

8. Vasselli JR, Yang JC, Linehan WM, White DE, Rosenberg SA, Walther MM (2001) Lack of retroperitoneal lymphadenopathy predicts survival of patients with metastatic renal cell carcinoma. J Urol 166(1):68-72

9. Capitanio U, Abdollah F, Matloob R et al (2013) When to perform lymph node dissection in patients with renal cell carcinoma: a novel approach to the preoperative assessment of risk of lymph node invasion at surgery and of lymph node progression during follow-up. BJU Int 112(2):E59-E66

10. Capitanio U, Suardi N, Matloob R et al (2013) Staging lymphadenectomy in renal cell carcinoma must be extended: a sensitivity curve analysis. BJU Int 111(3):412-418

11. Hutterer GC, Patard JJ, Perrotte P et al (2007) Patients with renal cell carcinoma nodal metastases can be accurately identified: external validation of a new nomogram. Int J Cancer 121(11):2556-2561

12. Gonen M, Schrag D, Weiser MR (2009) Nodal staging score: a tool to assess adequate staging of node-negative colon cancer. $\mathrm{J}$ Clin Oncol 27(36):6166-6171

13. Kluth LA, Abdollah F, Xylinas E et al (2013) Pathologic nodal staging scores in patients treated with radical prostatectomy: a postoperative decision tool. Eur Urol 66:439-446

14. Shariat SF, Rink M, Ehdaie B et al (2013) Pathologic nodal staging score for bladder cancer: a decision tool for adjuvant therapy after radical cystectomy. Eur Urol 63(2):371-378

15. Xylinas E, Rink M, Margulis V et al (2013) Prediction of true nodal status in patients with pathological lymph node negative upper tract urothelial carcinoma at radical nephroureterectomy. J Urol 189(2):468-473

16. Sobin L, Gospodariwicz M, Wittekind C (2009) TNM classification of malignant tumors. In: UICC International Union Against Cancer. pp 239-242. http://www.inen.sld.pe/portal/documentos/ pdf/educacion/13072015_TNM\%20Classification.pdf

17. Sobin LH, Wittekind CH (2002) TNM classification of malignant tumours. In: International Union Against Cancer (UICC). 6th edn. Wiley, New York, pp193-195. https://www.uicc.org/tnm-class ification-malignant-tumours-6th-edition

18. Fuhrman SA, Lasky LC, Limas C (1982) Prognostic significance of morphologic parameters in renal cell carcinoma. Am J Surg Pathol 6(7):655-663

19. Eble JN, Sauter G, Epstein JI, Sesterhenn IA (eds) (2004) World Health Organization classification of tumours. Pathology and genetics of tumours of the urinary system and male genital organs. IARC Press, Lyon

20. Janowitz T, Welsh SJ, Zaki K, Mulders P, Eisen T (2013) Adjuvant therapy in renal cell carcinoma-past, present, and future. Semin Oncol 40(4):482-491

21. Leibovich BC, Han KR, Bui MH et al (2003) Scoring algorithm to predict survival after nephrectomy and immunotherapy in patients with metastatic renal cell carcinoma: a stratification tool for prospective clinical trials. Cancer 98(12):2566-2575

22. Zisman A, Pantuck AJ, Wieder J et al (2002) Risk group assessment and clinical outcome algorithm to predict the natural history of patients with surgically resected renal cell carcinoma. J Clin Oncol 20(23):4559-4566

23. Meskawi M, Sun M, Trinh QD et al (2012) A review of integrated staging systems for renal cell carcinoma. Eur Urol 62(2):303-314

24. Terrone C, Guercio S, De Luca S et al (2003) The number of lymph nodes examined and staging accuracy in renal cell carcinoma. BJU Int 91(1):37-40

25. Joslyn SA, Sirintrapun SJ, Konety BR (2005) Impact of lymphadenectomy and nodal burden in renal cell carcinoma: retrospective analysis of the National Surveillance, Epidemiology, and End Results database. Urology 65(4):675-680

26. Sun M, Shariat SF, Cheng C et al (2011) Prognostic factors and predictive models in renal cell carcinoma: a contemporary review. Eur Urol 60(4):644-661 